\title{
FDA Device Listing Number
}

National Cancer Institute

\section{Source}

National Cancer Institute. FDA Device Listing Number. NCI Thesaurus. Code C101677.

A number assigned by the FDA to a device during Registration and Listing. 